\title{
Empfehlungen der Deutschen Atemwegsliga zur Spirometrie
}

\author{
Recommendations on Spirometry by Deutsche Atemwegsliga
}

Autoren

Institut
C.-P. Criée, D. Berdel, D. Heise, P. Kardos, D. Köhler, W. Leupold, H. Magnussen, W. Marek, R. Merget, H. Mitfessel, M. Rolke, S. Sorichter, W. Worth, H. Wuthe

Beatmungsmedizin/Schlaflabor, Bovenden-Lenglern
Bibliografie

DOI $10.1055 / s-2006-944245$ Pneumologie 2006; 60; 576-584 (c) Georg Thieme Verlag KG Stuttgart · New York ISSN 0934-8387

Korrespondenzadresse Prof. Dr. C.-P. Criée Ev. Krankenhaus GöttingenWeende $\cdot$ Abt. Pneumologie Pappelweg 5

37120 Bovenden-Lenglern criee@ekweende.de

\section{Einleitung}

Die Spirometrie ist eine einfache, schnelle, nichtinvasive und preisgünstige Untersuchung zur Messung von Lungenvolumina und Atemstromstärken. Ihr besonderer Wert liegt in der Diagnostik der sehr häufigen obstruktiven Ventilationsstörung und der Fähigkeit, deren therapeutische Beeinflussbarkeit zu objektivieren. In diesem Sinne dient sie zur Festlegung des Schweregrades obstruktiver Atmungserkrankungen und zur Beurteilung von Therapie, Krankheitsverlauf und Prognose. Aussagen über andere Störungen der Lungenfunktion, wie Gasaustausch oder Funktion der Atempumpe, sind nicht bzw. nur sehr eingeschränkt möglich. So können Patienten mit schwerster Ateminsuffizienz eine normale Spirometrie aufweisen. Mit der Spirometrie wird daher zwar ein sehr wichtiger, aber eben nur ein Teil der gesamten Lungenfunktion erfasst.

\section{Definition}

Unter Spirometrie versteht man die Messung von Lungenvolumina am Mund. Sie kann kontinuierlich zur Messung der Ventilation oder mittels willkürlicher Atemmanöver zur Bestimmung definierter Volumina und Atemstromstärken erfolgen. Die Messungen erfolgten früher mit einem Glocken- bzw. Trockenspirometer, heutzutage wird gewöhnlich ein Strömungs- bzw. Volumensensor verwendet.

Folgende Fragen kann die Spirometrie beantworten:

Liegt eine Atemwegsobstruktion vor?

Ist eine nachgewiesene Atemwegsobstruktion teilweise oder sogar vollständig reversibel (Reversibilitätstest mit Bronchodilatatoren)? Liegt eine relevante Verringerung der Lungenvolumina vor?

\section{Indikationen}

Dyspnoe (anfallsartig, unter Belastung, intermittierend)

Husten und/oder Auswurf

Screening (Gesundheitsuntersuchung)

Tabakkonsum

Verdacht auf Erkrankungen von Atemwegen, Lunge, Herz, knöchernem Thorax, Wirbelsäule, Skelettmuskulatur

Verdacht auf Erkrankungen der Atempumpe (Atemzentrum, zugehörige Nerven und Muskeln)

Verlaufsbeobachtung bronchopulmonaler Erkrankungen

Therapiekontrolle bronchopulmonaler Er-

krankungen

arbeitsmedizinische Überwachung (z.B.

Staubexposition, Rettungswesen)

präoperative Diagnostik.

\section{Kontraindikationen}

Spannungspneumothorax

akuter Herzinfarkt

akute innere Blutung.

\section{Messprinzip}

Üblicherweise werden heute offene Spirometer auf der Basis der Pneumotachographie verwendet, Glocken- bzw. Trocken-Spirometer sind technisch überholt. Bei der Pneumotachographie wird der flussproportionale Druckabfall an einem definierten Widerstand gemessen und daraus in Analogie zum Ohm'schen Gesetz der Atemfluss bestimmt. Anschließend wird durch Integration des Flusses über die Zeit das Atemvolumen berechnet. Sämtliche Parameter sind auf BTPS-Bedingungen (BTPS = body temperature 
pressure saturated) normiert, d.h. die erhobenen Daten gelten für $37 \mathrm{C}$ und $100 \%$ relative Feuchte beim gegebenen Luftdruck. Exspiratorische Lungenvolumina und Strömungen werden demzufolge direkt erfasst, während inspiratorische Größen, für die die ATP-Umgebungsbedingungen gelten (ATP = ambient temperature pressure) auf BTPS korrigiert werden müssen. Ein Spirometer muss mit einfachen Mitteln durch Kalibrierung überprüfbar sein. Erforderlich ist die tägliche Kalibrierung, wobei das Kalibriervolumen (= Pumpenhub von 1-3 Liter) mit einem Fehler von unter $0,5 \%$ bestimmt werden muss (Ausnahme: Spirometer, die auf der Ultraschall-Laufzeitmessung basieren). Eine Überprüfung der Kalibrierung ist zusätzlich durchzuführen, wenn trotz guter Mitarbeit des Untersuchten und nach Überprüfung der persönlichen Daten ein Ergebnis trotz Austausch der Sensorik bzw. Reinigung des Systems nicht plausibel erscheint. Sinnvoll ist auch die gelegentliche Überprüfung der Kalibrierung anhand der bekannten Lungenvolumina eines Mitarbeiters. Die Ergebnisse der Kalibrierung sind in einem Gerätebuch zu dokumentieren.

\section{Messparameter}

Man unterscheidet zwischen statischen und dynamischen Lungenvolumina. Unter den statischen Lungenvolumina versteht man Lungenvolumina, deren Messwerte nicht vom zeitlichen Ablauf des Spirogramms abhängen, z. B. Vitalkapazität (VC). Unter dynamischen Lungenvolumina versteht man Lungenvolumina, deren Messwerte vom zeitlichen Verlauf abhängig sind z. B. Einsekundenkapazität $\left(\mathrm{FEV}_{1}\right)$. Da offene Spirometer primär die Atemstromstärke messen, können zusätzlich zu den Lungenvolumina problemlos auch spezielle Atemstromstärken bestimmt werden ( Abb. 1). Die wichtigsten Parameter sind in Tab. 1 aufgeführt.

\section{Vitalkapazitåt (VC)}

Die Vitalkapazität (VC) ist die Volumendifferenz, die am Mund zwischen vollständiger Inspiration (zur Totalkapazität TLC) und vollständiger Exspiration (zum Residualvolumen RV) gemessen werden kann. In Deutschland und einigen europäischen Ländern wird sie als „inspiratorische Vitalkapazität“ IVC bestimmt. Aus normaler Ruheatmung heraus wird langsam bis zum RV ausgeatmet und anschließend zügig - aber nicht forciert - bis zur TLC eingeatmet. In Großbritannien und Nordamerika wird die Vitalkapazität auch während einer langsamen („relaxed“) Exspiration vom TLC-Niveau aus mit ansteigender Anstrengung am Exspirationsende (EVC), in der Regel aber während einer forcierten Exspiration (FVC) gemessen. Bei gesunden Probanden besteht keine systematische Differenz zwischen IVC und EVC, nur bei obstruktiven Lungenerkrankungen kann die IVC größer sein als EVC und FVC; EVC ist in der Regel größer als FVC.

\section{Dynamische Lungenvolumina und maximale exspiratorische Atemstromstärken}

Als forcierte Exspiration wird das Manöver bezeichnet, bei dem der Proband zügig bis zur TLC einatmet und sofort danach mit maximaler Anstrengung über mehrere Sekunden bis zum Residualvolumen ausatmet. Die maximale Muskelkraft soll dabei „schlagartig“ und nicht allmählich aufgebaut werden. Wird die forcierte Exspiration verzögert begonnen, sollte der Versuch wiederholt werden. Bei diesem - auch Tiffeneau-Test genannten - Manöver werden das exspirierte Volumen in der ersten Sekunde (forciertes Exspirationsvolumen, $\mathrm{FEV}_{1}$ ) sowie die maximalen exspiratorischen Atemstromstärken bestimmt. Dabei wird sowohl das Volumen gegen die Zeit aufgetragen (Volumen/ZeitKurve), als auch die Atemstromstärke gegen das Lungenvolumen (Fluss/Volumen-Kurve) ( Abb. 1). Diese Darstellung sollte routinemäßig gewählt werden.

Bei dem forcierten Exspirationsmanöver sind 2 Phänomene zu berücksichtigen:

Die Anstrengungsabhängigkeit („effort-dependence“): Bei maximaler exspiratorischer Anstrengung sind die maximalen exspiratorischen Atemstromstärken durch die Kompression der Atemwege häufig geringer als bei submaximaler Anstrengung, was sich insbesondere bei Patienten mit obstruktiver Ventilationsstörung auswirkt. Da aber die Atemstromstärken bei submaximaler Anstrengung schlecht reproduzierbar sind, wird das maximal forcierte Manöver bevorzugt. Die Zeitabhängigkeit („time-dependence“): Bei langsamer Inspiration mit langer Pause vor der forcierten Exspiration

Tab. 1 Spirometrische Parameter

\begin{tabular}{|c|c|c|c|}
\hline Parameter & Definition & Symbol & Einheit \\
\hline inspiratorische Vitalkapazität & $\begin{array}{l}\text { Atemvolumen, welches nach kompletter Exspiration } \\
\text { maximal eingeatmet werden kann }\end{array}$ & IVC (Synonym: VC in) & 1 \\
\hline forcierte Vitalkapazität & $\begin{array}{l}\text { Atemvolumen, welches nach kompletter Inspiration } \\
\text { forciert maximal ausgeatmet werden kann }\end{array}$ & FVC & 1 \\
\hline $\begin{array}{l}\text { forciertes exspiratorisches Volumen } \\
\text { in } 1 \text { Sekunde, Einsekundenkapazität }\end{array}$ & $\begin{array}{l}\text { Atemvolumen, welches nach maximaler Inspiration } \\
\text { forciert in der ersten Sekunde ausgeatmet werden } \\
\text { kann }\end{array}$ & $\mathrm{FEV}_{1}$ & 1 \\
\hline $\begin{array}{l}\text { relative Einsekundenkapazität, } \\
\text { Tiffeneau-Index }\end{array}$ & $\begin{array}{l}\text { forciertes exspiratorisches Volumen in } 1 \text { Sek., } \\
\text { ausgedrückt in \% der inspiratorischen Vitalkapazität }\end{array}$ & $\mathrm{FEV}_{1} / \mathrm{IVC}$ & $\%$ \\
\hline $\begin{array}{l}\text { maximaler exspiratorischer } \\
\text { Spitzenfluss, „Peak flow“ }\end{array}$ & $\begin{array}{l}\text { Spitzenfluss bei maximaler exspiratorischer } \\
\text { Anstrengung }\end{array}$ & PEF & $1 \times \mathrm{s}^{-1 * *}$ \\
\hline $\begin{array}{l}\text { maximaler exspiratorischer Fluss } \\
\text { bei } 50 \% \text { der FVC }\end{array}$ & $\begin{array}{l}\text { maximale Atemstromstärke nach Ausatmung } \\
\text { von } 50 \% \text { der FVC }\end{array}$ & $\mathrm{MEF}_{50 \%}$, Synonym FEF $50 \%$ & $1 \times s^{-1}$ \\
\hline $\begin{array}{l}\text { maximaler exspiratorischer Fluss } \\
\text { bei } 25 \% \text { der FVC }\end{array}$ & $\begin{array}{l}\text { maximale Atemstromstärke nach Ausatmung } \\
\text { von } 75 \% \text { der FVC }\end{array}$ & $\mathrm{MEF}_{25 \%}$, Synonym $\mathrm{FEF}_{75 \%}$ & $1 \times s^{-1}$ \\
\hline
\end{tabular}




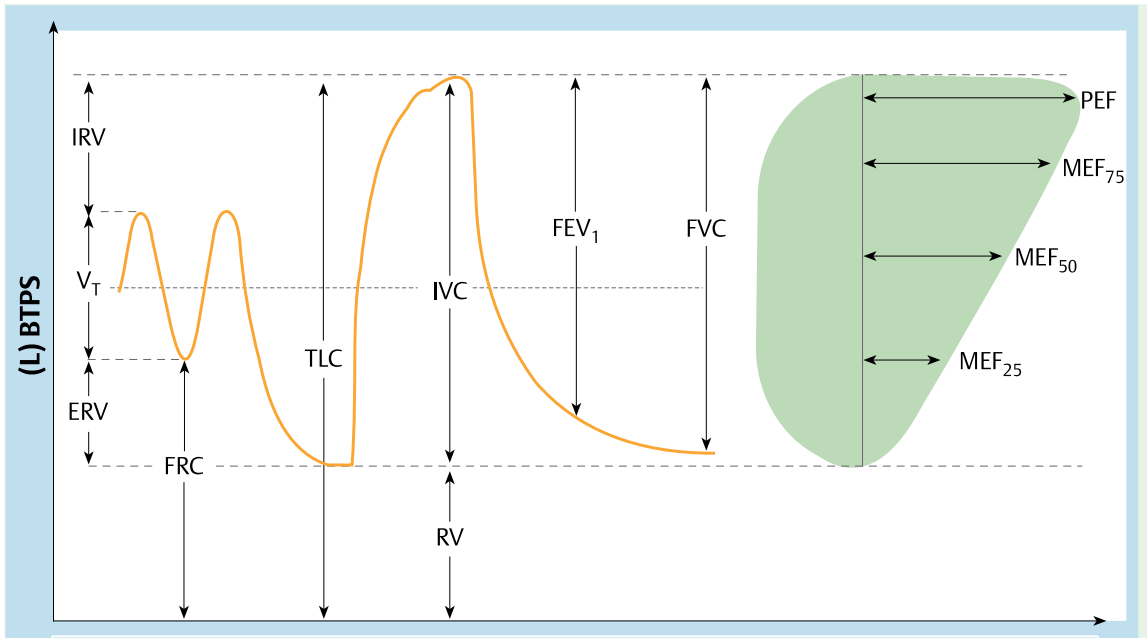

Abb. 1 Mobilisierbare und nicht mobilisierbare Lungenvolumina, sowie maximale exspiratorische Flüsse. Linke Bildhälfte: Volumen/Zeit-Kurve. Rechte Bildhälfte: Fluss/Volumen-Kurve.

Mobilisierbare und nicht mobilisierbare Lungenvolumina, sowie maximale exspiratorische Flüsse. IRV = inspiratorisches Reservevolumen (L), ERV=exspiratorisches Reservevolumen (L),

$\mathrm{V}_{\mathrm{T}}=$ Atemzugvolumen $(\mathrm{L}), \mathrm{FRC}=$ Funktionelle Residualkapazität $(\mathrm{L}), \mathrm{TLC}=$ Totale Lungenkapazität $(\mathrm{L})$, IVC = inspiratorische Vitalkapazität (L), RV=Residualvolumen $(\mathrm{L})$,

$\mathrm{FEV}_{1}=$ forciertes exspiratorisches Volumen in 1 Sekunde (L), FVC= forcierte Vitalkapazität (L),

$\mathrm{PEF}=$ exspiratorischer Spitzenfluss (L/S).

$\mathrm{MEF}_{75^{\prime} 50^{\prime} 25^{\prime}}=$ maximale exspiratorische Flüsse als Prozent der FVC (L/s).

vom TLC-Niveau aus sind die Atemstromstärken und die FEV 1 bis zu 25\% geringer als bei schneller Inspiration ohne Pause vor der forcierten Exspiration. Die höheren Flüsse bei forcierter Exspiration ohne Pause nach der Inspiration kommen durch die viskoelastischen Eigenschaften der Lunge nach vorausgegangener Dehnung zustande, aber auch durch eine bessere Aktivierung der Exspirationsmuskulatur. Es wird daher eine zügige Inspiration mit nur kurzer Pause (<1 Sek.) mit anschließender forcierter Exspiration empfohlen. Dies ist wichtig zu beachten, da die unterschiedliche Ausführung des Manövers beim Bronchodilatationstest zu falsch-positiven oder falsch-negativen Ergebnissen führen kann.

\section{Parameter zur Erfassung der forcierten Exspiration}

\section{Einsekundenkapazität, forciertes exspiratorisches Volumen in der ersten Sekunde $\left(\mathrm{FEV}_{1}\right)$}

Das Volumen, welches nach maximaler Inspiration mittels forcierter Exspiration in der 1. Sekunde ausgeatmet werden kann, wird als (absolute) Einsekundenkapazität $\left(\mathrm{FEV}_{1}\right)$ bezeichnet. Die Einsekundenkapazität in Prozent der Vitalkapazität wird Tiffeneau-Index oder relative Einsekundenkapazität genannt. In Deutschland und Frankreich wird üblicherweise die inspiratorische Vitalkapazität IVC in den Nenner gesetzt, hingegen im nordamerikanischen Raum zumeist die forcierte Vitalkapazität FVC.

\section{Spitzenfluss (PEF)}

Der Spitzenfluss PEF (Peak Expiratory Flow) ist die maximal erreichbare Atemstromstärke bei forcierter Exspiration. Er kann an der Fluss/Volumen-Kurve unmittelbar abgelesen werden. Das Messergebnis hängt wie bei allen Atemstromstärken stark von der Mitarbeit der Patienten ab.

Da der PEF gut mit dem Schweregrad der obstruktiven Ventilationsstörung einer asthmatischen Erkrankung korreliert, wird er zur Verlaufs- bzw. Therapiekontrolle genutzt. Hierzu misst der
Patient mithilfe eines Peakflow-Meters mehrmals täglich den PEF zu Hause, insbesondere bei akuter Atemnot oder Instabilität der Erkrankung. Es ist zu beachten, dass Peakflow-Meter ihre Messergebnisse in $\mathrm{L} \times \mathrm{min}^{-1}$ und nicht in $\mathrm{L} \times \mathrm{s}^{-1}$ anzeigen. Außerdem ist das Atemmanöver bei alleiniger Bestimmung des PEF nicht identisch mit der oben beschriebenen forcierten Exspiration über mehrere Sekunden.

\section{Maximale exspiratorische Flüsse bei $x \%$ der Vitalkapazität ( $\mathrm{FEF}_{\mathrm{x}} \%$ bzw. $\mathrm{MEF}_{\mathrm{x}} \%$ )}

Trägt man in die Fluss/Volumen-Kurve die Volumengrenzen ein, bei der jeweils ein Viertel der forcierten Vitalkapazität ausgeatmet wurde, so kann man an diesen Stellen die zugehörigen maximalen Atemstromstärken ablesen: Auf diese Weise erhält man Werte für die maximalen exspiratorischen Flüsse $\mathrm{MEF}_{75}, \mathrm{MEF}_{50}$ und $\mathrm{MEF}_{25}$ (jeweils bezogen auf den Prozentsatz der FVC, der dann noch ausgeatmet werden kann!). In der neuesten amerikanisch-europäischen Empfehlung wird die maximale exspiratorische Atemstromstärke (MEF) als FEF (forced expiratory flow) angegeben. Der Index ist das Volumen, welches bereits ausgeatmet ist, so ist z. B. $\mathrm{FEF}_{75}$ nach dieser Empfehlung identisch mit der oben definierten $\mathrm{MEF}_{25}$. Es wird auch nicht mehr empfohlen die Volumengrenzen anhand der inspiratorischen Vitalkapazität festzulegen. Die maximalen exspiratorischen Flüsse sind sehr empfindliche Indikatoren für jede Art von Veränderungen in den kleinen Atemwegen. Der Bezug auf die jeweilige FVC schmälert allerdings ihre Verwendbarkeit für die Beurteilung des bronchodilatatorischen Effektes. Bei Patienten nach Transplantation kann ein im Verlauf abnehmender $\mathrm{MEF}_{25}$-Wert ( $\mathrm{FEF}_{75}$-Wert) ein Hinweis auf eine Abstoßungsreaktion sein.

Die mittlere maximale (forcierte) exspiratorische Atemstromstärke, die zwischen 25 und 75\% der forcierten Vitalkapazität ausgeatmet wird, wird als MMEF oder FEF 25 - 75\% bezeichnet. Dieser Parameter gilt als sehr sensibel zur Erkennung einer beginnenden Atemwegsobstruktion, ein Vorteil gegenüber der Bestimmung von $F F_{50}$ oder $F E F_{75}$ (bzw. MEF 50 oder $M E F_{25}$ ) ist aber nicht belegt. 
Tab. 2 Kriterien für eine repräsentative forcierte Exspiration

\section{Reproduzierbarkeitskriterien:}

Die Differenz zwischen größtem und zweitgrößtem Wert darf bei FEV $_{1}$ nicht mehr als $5 \%$ betragen * FVC nicht mehr als $5 \%$ betragen* PEF nicht mehr als $10 \%$ betragen

${ }^{*}$ Bei FVC $<1$ I darf die Differenz nicht mehr als 100 ml betragen. Akzeptanzkriterien:

Der maximale exspiratorische Spitzenfluss („Peakflow“ PEF) soll innerhalb von 120 ms erreicht werden (steiler Anstieg).

keine Artefakte (Husten, Glottisschluss, Leckagen, vorzeitige Beendigung, unterschiedliche Anstrengung)

die Exspiration ist erst beendet, wenn der kontinuierliche Fluss unter $0,1 \mid \times s^{-1}$ abgesunken ist.

Tab. 3 Dokumentation Mitarbeit und technische Qualität

\begin{tabular}{|c|c|}
\hline Mitarbeit & technische Qualität \\
\hline einwandfrei & Messung fehlerhaft \\
\hline gut & Messung ohne relevante Fehle \\
\hline $\begin{array}{l}\text { eingeschränkt wegen mangeln- } \\
\text { dem Verständnis }\end{array}$ & Messung noch brauchbar \\
\hline eingeschränkt wegen Hustenreiz & Messung teilweise fehlerhaft \\
\hline eingeschränkt wegen Angst & Messung mit großen Fehlern \\
\hline \multicolumn{2}{|l|}{$\begin{array}{l}\text { eingeschränkt wegen mangeln- } \\
\text { der Kraft }\end{array}$} \\
\hline \multicolumn{2}{|l|}{ eingeschränkt wegen Schmerzen } \\
\hline $\begin{array}{l}\text { eingeschränkt wegen mangeln- } \\
\text { der Bereitschaft }\end{array}$ & \\
\hline
\end{tabular}

Das Volumen, welches bei einer forcierten Inspiration vom Residualvolumen in 1 Sekunde eingeatmet wird, wird als FIV bezeichnet. Dieses forcierte Inspirationsvolumen in 1 Sekunde beträgt bei gesunden Probanden ca. 95\% der inspiratorischen forcierten Vitalkapazität. Dieses Volumen ist ein Maß für die inspiratorisch wirksame Obstruktion und für die inspiratorisch wirksame Muskelkraft. Es ist kein Standardparameter der Spirometrie.

Die Inspiratorische Kapazität (IC) ist das Volumen zwischen FRC und TLC ( Abb. 1). Nach mehreren Atemzügen und stabilem endexspiratorischen Volumen erfolgt die zügige maximale Einatmung bis zur TLC. Es sollte der Mittelwert aus 3 Manövern zur Auswertung kommen. Die inspiratorische Kapazität ist ein direktes Maß zur Abschätzung der Lungenüberblähung, ihre Verminderung auf unter 25\% der TLC ist mit einer ungünstigen Prognose verbunden. Sie wird auch zur Abschätzung einer Änderung der FRC durch Pharmaka oder Belastung genutzt.

\section{Durchführung der Untersuchung}

Der Patient sollte beengende Kleidungsstücke ablegen. Die Messung wird im Sitzen durchgeführt, da sich alle Normalwerte auf die sitzende Position beziehen.

Die Nase wird mit einer Nasenklemme luftdicht verschlossen. Der Patient nimmt das Mundstück vor dem Pneumotachographen zwischen die Zähne, die Zunge liegt unter dem Mundstück.

Nachdem einige Male ruhig ein- bzw. ausgeatmet wurde, wird er aufgefordert, langsam pressend maximal auszuatmen. Danach erfolgt eine zügige vollständige Inspiration zur Bestimmung der inspiratorischen Vitalkapazität.
An dieses Manöver schließt sich nach einer möglichst geringen Pause (unter $1 \mathrm{sec}$ ) eine forcierte maximale Exspiration an. Der Patient muss dazu angehalten werden, das minimale bzw. das maximale Lungenvolumen (also erst RV, dann TLC und wieder RV) wirklich zu erreichen. Um das RV bei langsamer und vor allem bei forcierter Exspiration möglichst gut zu erreichen, kommt es darauf an, so lange wie möglich auszuatmen, bis ein deutliches Plateau im zeitlichen Volumenverlauf sichtbar wird. Dies fällt vor allem Patienten mit einer obstruktiven Ventilationsstörung schwer.

\section{Kriterien für eine akzeptable Durchführung der Messung}

Die Akzeptanzkriterien sind in Tab. 2 zusammengestellt. Das jeweilige Exspirationsmanöver ist korrekt beendet, wenn der kontinuierliche Fluss unter $0,11 \times \mathrm{s}^{-1}$ abgesunken ist. Andere Zeitkriterien, wie z.B. eine Exspiration von mindestens 4 Sekunden, sind z. B. für Kinder und Patienten mit Lungengerüsterkrankung nicht möglich und sollten nicht verwendet werden. Um die Reproduzierbarkeit (auch „repeatability“, also „Wiederholbarkeit“" genannt) - und damit die Güte der Mitarbeit - bestimmen zu können, müssen mindestens 3 Versuche durchgeführt werden, wobei sich die Ergebnisse der besten 2 Versuche für $\mathrm{FEV}_{1}$ und FVC um weniger als $5 \%$ (bei einer FVC $<11$ weniger als $100 \mathrm{ml}$ ) und für PEF um weniger als $10 \%$ unterscheiden dürfen. Hohe Reproduzierbarkeit ist trotz guter Mitarbeit nicht erreichbar, wenn durch die forcierten Manöver ein „SpirometerAsthma“ induziert wurde. Diese Komplikation ist bei 2 - 3 Versuchen selten, die Wahrscheinlichkeit steigt aber mit der Zahl der Versuche, weil jede forcierte Exspiration eine Art von Provokationstest darstellt: Mehr als 4 forcierte Exspirationen sind nicht sinnvoll. Verstöße gegen die Akzeptanzkriterien müssen dokumentiert werden (soweit möglich automatisch von der MessSoftware) und die vom Untersucher abgeschätzte Güte der Mitarbeit muss auf dem Untersuchungsprotokoll notiert werden (Muster in Tab. 3) (๑ Abb. 2).

\section{Auswertung}

Die höchsten Werte für IVC, $\mathrm{FEV}_{1}$ und FVC werden aus allen Manövern ermittelt, die die o.g. Akzeptanz- und Reproduzierbarkeitskriterien erfüllen. Die maximalen exspiratorischen Atemstromstärken werden aus der optisch besten Fluss/VolumenKurve bzw. aus der mit der größten Summe aus FEV 1 und FVC bestimmt. Wahrscheinlich wird die „beste“ Kurve durch die höchste Summe von FVC, FEV $V_{1}$ und 1/3 $\times$ PEF beschrieben, dies ist jedoch nicht validiert.

Diese Kurve ist numerisch und graphisch zu dokumentieren. Um auch die Reproduzierbarkeit zu dokumentieren, bieten sich 2 Möglichkeiten an: Man notiert die Reproduzierbarkeit numerisch oder/und man dokumentiert alle akzeptablen Fluss/Volumen-Kurven grafisch. Die grafische Darstellung muss jederzeit eine erneute Formanalyse ermöglichen.

Nicht empfohlen wird die so genannte Hüllkurve. Dabei werden die Fluss/Volumen-Kurven am Ausgangspunkt der forcierten Exspiration (TLC) übereinandergelegt und die Maximalkurve konstruiert, die Maximalflüsse werden dann von der Maximalkurve abgelesen. Die Maximalkurve ist daher eine konstruierte, fiktive Kurve, die nie geatmet wurde. Typische Fehlerzeichen bei der 
Tab. 4 Regressionsgleichungen (EGKS-Werte) für Lungenvolumina und exspiratorische Atemstromstärken für Erwachsene im Alter von 18 - 70 Jahren

\begin{tabular}{|c|c|c|c|c|}
\hline & & & Mittelwert-Gleichung & $1,64 \times \mathrm{RSD}$ \\
\hline \multirow[t]{7}{*}{ Männer } & IVC & (I) & $6,10 \mathrm{KG}-0,028 \mathrm{~A}-4,65$ & $\pm 0,92$ \\
\hline & FVC & (I) & $5,76 \mathrm{KG}-0,026 \mathrm{~A}-4,34$ & $\pm 1,00$ \\
\hline & $\mathrm{FEV}_{1}$ & (I) & $4,30 \mathrm{KG}-0,029 \mathrm{~A}-2,49$ & $\pm 0,84$ \\
\hline & PEF & $\left(1 \times s^{-1}\right)$ & $6,14 \mathrm{KG}-0,043 \mathrm{~A}+0,15$ & $\pm 1,99$ \\
\hline & $\mathrm{FEF}_{50 \%}=\mathrm{MEF}_{50 \%}$ & $\left(1 \times s^{-1}\right)$ & $3,79 \mathrm{KG}-0,031 \mathrm{~A}-0,35$ & $\pm 2,17$ \\
\hline & $\mathrm{FEF}_{75 \%}=\mathrm{MEF}_{25 \%}$ & $\left(1 \times \mathrm{s}^{-1}\right)$ & $2,61 \mathrm{KG}-0,026 \mathrm{~A}-1,34$ & $\pm 1,28$ \\
\hline & $\mathrm{FEV}_{1} / \mathrm{VC}(\%)$ & & $-0,18 A+87,21$ & $\pm 11,8$ \\
\hline \multirow[t]{7}{*}{ Frauen } & IVC & (I) & $4,66 \mathrm{KG}-0,024 \mathrm{~A}-3,28$ & $\pm 0,69$ \\
\hline & FVC & (I) & $4,43 \mathrm{KG}-0,026 \mathrm{~A}-2,89$ & $\pm 0,71$ \\
\hline & $\mathrm{FEV}_{1}$ & (I) & $3,95 \mathrm{KG}-0,025 \mathrm{~A}-2,60$ & $\pm 0,62$ \\
\hline & PEF & $\left(1 \times s^{-1}\right)$ & $5,50 \mathrm{KG}-0,030 \mathrm{~A}-1,11$ & $\pm 1,48$ \\
\hline & $\mathrm{FEF}_{50 \%}=\mathrm{MEF}_{50 \%}$ & $\left(1 \times s^{-1}\right)$ & $2,45 \mathrm{KG}-0,025 \mathrm{~A}+1,16$ & $\pm 1,81$ \\
\hline & $\mathrm{FEF}_{75 \%}=\mathrm{MEF}_{25 \%}$ & $\left(1 \times s^{-1}\right)$ & $1,05 \mathrm{KG}-0,025 \mathrm{~A}+1,11$ & $\pm 1,13$ \\
\hline & $\mathrm{FEV}_{1} / \mathrm{VC}(\%)$ & & $-0,19 A+89,10$ & $\pm 10,7$ \\
\hline
\end{tabular}

$\mathrm{KG}=$ Körperlänge in $\mathrm{m}, \mathrm{A}=$ Alter in Jahren

Zwischen 18 und 25 Jahren wird in die Sollwertgleichung das Alter 25 eingesetzt. Die 5. Perzentile errechnet sich durch Subtraktion von 1,64 × RSD

(residuale Standardabweichung) vom errechneten Mittelwert (z. B. ist der untere Grenzwert für IVC bei Männern: Mittlere IVC-0,92 I)

Sollwerte für Kinder in: Lindemann H., W. Leupold: Lungenfunktionsdiagnostik bei Kindern. Kohlhammer, Stuttgart 2003.

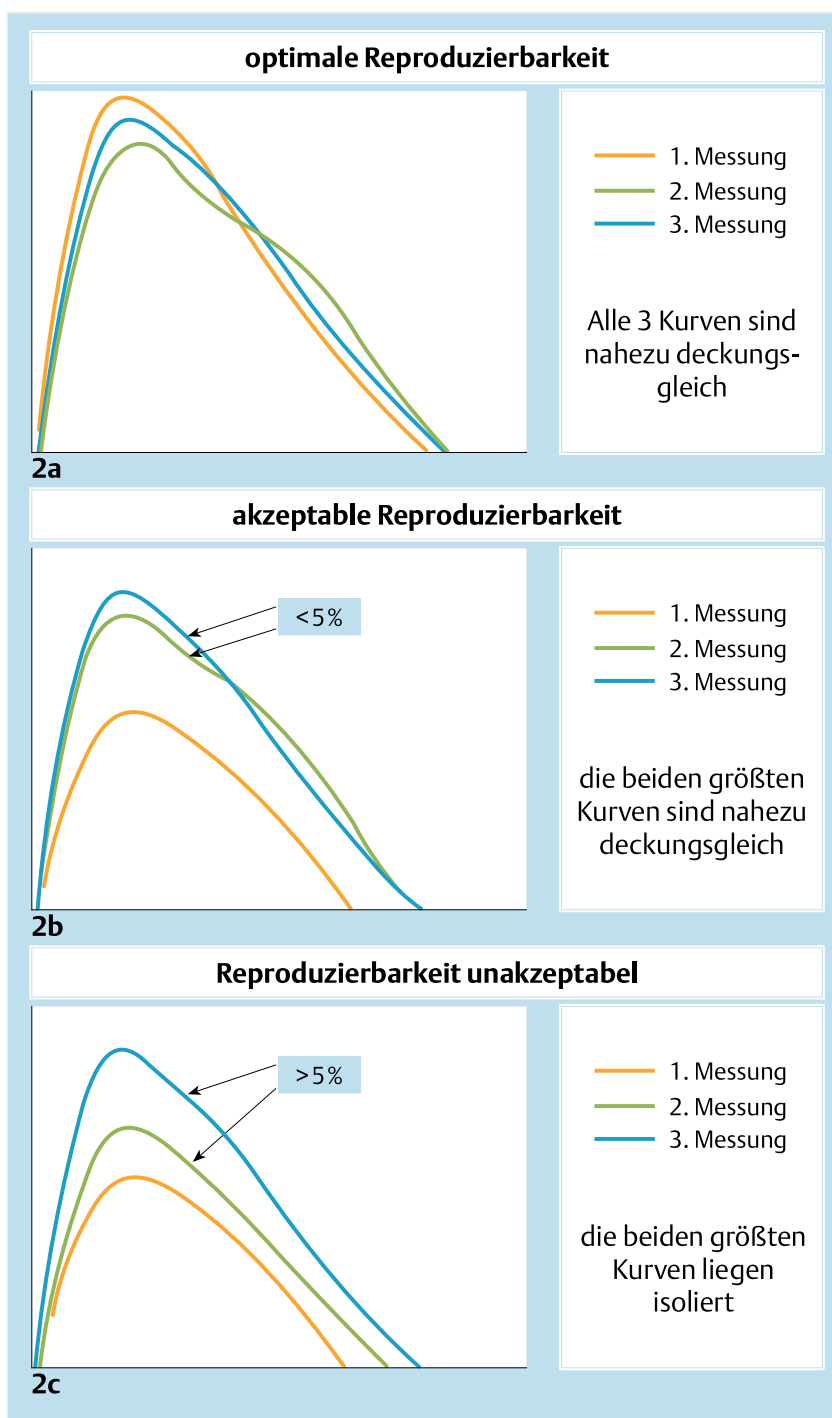

Abb. 2 Reproduzierbarkeitskriterien.
Patienten-Mitarbeit bei der Auswertung der Fluss/VolumenKurve zeigt die $\bullet$ Abb. 3.

\section{Normalwerte}

Die gebräuchlichsten Normalwerte sind von der European Respiratory Society (ERS) zuletzt 1993 publiziert worden. Es handelt sich um die so genannten EGKS (Europäische Gesellschaft für Kohle und Stahl)-Werte. Die Regressionsgleichungen zur Berechnung des Sollwertes sind in Tab. 4 angegeben, die Streuung ist im Wesentlichen auf die lockere Korrelation zwischen Körperlänge und Lungenvolumen zurückzuführen. Werte unterhalb der 5. Perzentile des Frequenzspektrums der in der Referenzpopulation gemessenen Werte, also des „Normalbereichs“, gelten als pathologisch mit einer Irrtumswahrscheinlichkeit von unter $5 \%$. Anders ausgedrückt weisen weniger als $5 \%$ der gesunden Bevölkerung einen Wert unterhalb der 5. Perzentile auf. Die 5. Perzentile wird durch Subtraktion von 1,64 $\times$ RSD (residuale Standardabweichung, Restvarianz) vom errechneten Sollwert ermittelt (Tab. 4). Somit sind aber auch bis zu 5\% der unterhalb des Normbereichs liegenden Werte nicht krankheitsbedingt. Auch kann ein im Normbereich liegender Wert pathologisch sein, so kann z.B. eine im Normbereich liegende Vitalkapazität durch einen Lungengerüstprozess von einem zuvor deutlich höheren Wert reduziert worden sein oder eine im Normbereich liegende $\mathrm{FEV}_{1}$ durch einen Bronchodilatationstest ansteigen. Patienten können eine klinisch manifeste COPD aufweisen, obwohl ihre spirometrischen Werte noch im Normbereich liegen. Darum sollte man den individuellen Verlauf der Kenngrößen der Lungenfunktion verfolgen, um beurteilen zu können, ob die erhobenen Werte für den individuellen Patienten noch normal oder schon pathologisch sind.

\section{Bewertung der Spirometrie}

Mittels Spirometrie können unterschiedliche Ventilationsstörungen nachgewiesen und quantifiziert werden. Problemlos gelingt dies für die obstruktive Ventilationsstörung, für die restrik- 


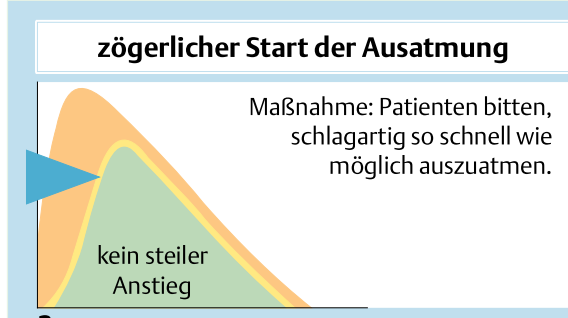

$3 a$

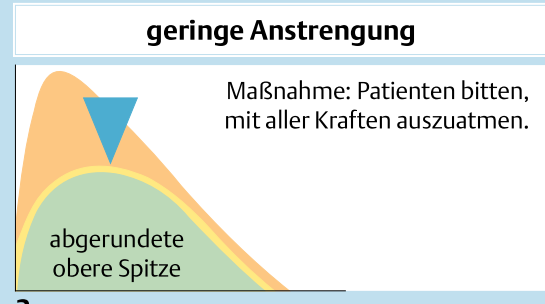

$3 c$

unvollständige Ausatmung und frühzeitiger Abbruch der Ausatmung

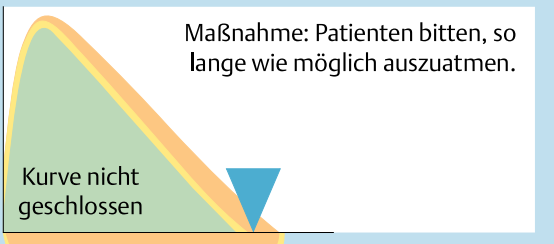

$3 e$

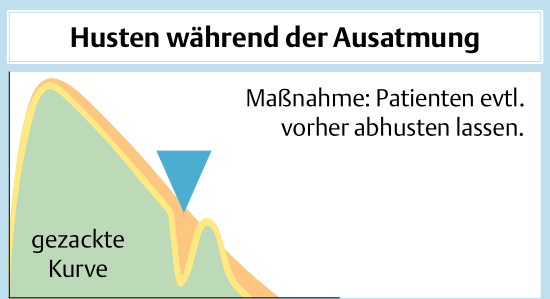

3b

vorher nicht maximal eingeatmet

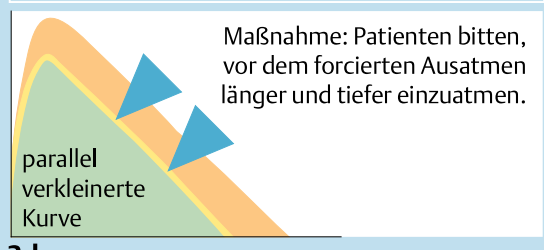

$3 d$

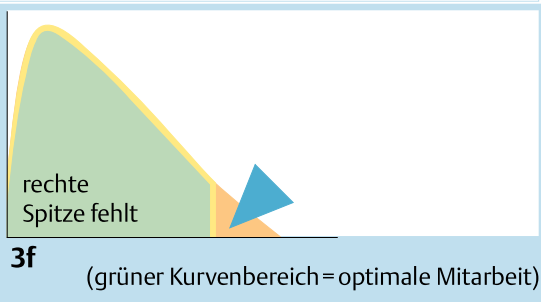

Abb. 3 Fehlerzeichen, die an eine mangelhafte Mitarbeit des Patienten hinweisen. tive Ventilationsstörung allerdings nur mit erheblichen Einschränkungen.

\section{Obstruktive Ventilationsstörung}

Eine obstruktive Ventilationsstörung ist durch eine Verminderung des altersabhängigen Tiffeneau-Index $\left(\mathrm{FEV}_{1} / \mathrm{IVC}\right)$ auf Werte unterhalb der 5. Perzentile definiert. Dabei ist in der Regel auch die absolute Einsekundenkapazität kleiner als der Normalwert. Charakteristisch ist die Abnahme der maximalen exspiratorischen Atemstromstärken. Der spirometrische Schweregrad der obstruktiven Ventilationsstörung ergibt sich aus der Einschränkung der $\mathrm{FEV}_{1}$, ausgedrückt in Prozent des Sollwerts ( Tab. 5a). Er muss nicht mit dem klinischen Schweregrad der Erkrankungen wie COPD oder Asthma übereinstimmen. Außerdem sind die Spirometriedaten nur ein Teil der Surrogatparameter, die die Beurteilung des klinischen Schweregrades von Asthma und COPD bestimmen. So sind z. B. bei Asthma auch die Symptomatik tagsüber und nachts sowie die PEF-Schwankungen zu berücksichtigen. Selbst bei präbronchodilatatorisch gemessener normaler Spirometrie kann somit ein mittelschweres persistierendes Asthma vorliegen, wenn der Patient nächtliche Anfälle hat ( Tab.5b). Bei der Schweregradeinteilung der COPD wird die postbronchodilatatorische $\mathrm{FEV}_{1}$ gewählt, um die chronische (scheinbar irreversible) Lungenfunktionseinschränkung als Grundlage der Beurteilung heranzuziehen, aber es werden auch die Blutgase berücksichtigt ( Tab. 5c). Häufig ist die Vitalkapazität aufgrund einer zusätzlich bestehenden Lungenüberblähung vermindert, wobei aber die Totale Lungenkapazität (TLC) normal oder erhöht ist. Erkrankungen, die mit einer obstruktiven Ventilationsstörung einhergehen, sind in Tab. 5a aufgeführt. Stenosen in den oberen Atemwegen (z.B. tumorbedingte Trachealstenosen oder Stimmbandparesen) können zur Plateau- bildung in der Fluss/Volumen-Kurve führen, d.h. große Atemströme werden regelrecht gekappt. Bei begründetem Verdacht auf eine zentrale Atemwegsstenose (Mundstück? Gebissposition? Stridor!) sollte nicht nur die forcierte exspiratorische, sondern auch die forcierte inspiratorische Fluss/Volumen-Kurve bestimmt werden, die ebenfalls eine Limitierung der Spitzenflüsse zeigt. Da die Strömungshindernisse oft mechanisch instabil sind, sind die Fluss/Volumen-Kurven dann allerdings schlecht reproduzierbar. Bei dem optischen Eindruck einer exspiratorischen Plateaubildung in der Fluss/Volumen-Kurve muss an einen Tu-

\section{Tab. 5a Obstruktive Ventilationsstörung}

\section{Definition}

$\mathrm{FEV}_{1} /$ IVC $<5$. Perzentile des Sollwertes Schweregrade

$\begin{array}{lll}\text { I } & \text { leicht } & \mathrm{FEV}_{1}>70 \% \text { Soll } \\ \text { II } & \text { mäßig } & \mathrm{FEV}_{1} 60-69 \% \text { Soll } \\ \text { III } & \text { mittelschwer } & \mathrm{FEV}_{1} 50-59 \% \text { Soll } \\ \text { IV } & \text { schwer } & \mathrm{FEV}_{1} 35-49 \% \text { Soll } \\ \text { V } & \text { sehrschwer } & \mathrm{FEV}_{1}<35 \% \text { Soll }\end{array}$

Der spirometrische Schweregrad der obstruktiven Ventilationsstörung stimmt nicht mit dem Schweregrad der Erkrankung, z. B. Asthma oder COPD, überein (s. Tab. 5 b, c)

Differenzialdiagnosen

Asthma bronchiale COPD Bronchiektasie zystische Fibrose (Mukoviszidose) Silikose Stenose im Bereich der großen Atemwege (cave: Tumor) Lungenparenchymerkrankungen mit Obstruktion (z. B. Sarkoidose) 


\section{Symptome tagsüber}

4

schwergradig

3

mittelgradig

persistierend

2

persistierend

1

intermittierend persistierend
Dauersymptome

eingeschränkte körperliche Aktivität, hohe Intensität + Variabilität

täglich

$>1 \mathrm{mal} /$ Woche

$<1 \mathrm{mal} / \mathrm{Tag}$

$<1$ mal/Woche

dazwischen asymptomatisch nächtliche Symptome

häufig

$>1 \mathrm{mal} /$ Woche

$>2 \mathrm{mal} /$ Monat

$\leq 2 \mathrm{mal} /$ Monat
Ein einziges Kriterium eines höheren Schweregrades ist bereits ausreichend, den Patienten dem höheren Schweregrad zuzuordnen.

Für die Beurteilung des Schweregrades des Asthmas sollte die prädilatatorische FEV ${ }_{1}$ gemessen werden. Berechnung der zirkadianen PEF-Variabilität: Geringster PEF-Morgenwert bei Messungen über 7 Tage in \% des PEF-Bestwertes.

\section{FEV $_{\mathbf{1}}$ oder PEF}

$\leq 60 \%$ vom Sollwert

PEF-Variabilität > $30 \%$

$60-80 \%$ vom Sollwert

PEF-Variabilität

$20-30 \%$

$\geq 80 \%$ vom Sollwert

PEF-Variabilität

$20-30 \%$

$\geq 80 \%$ vom Sollwert PEF-Variabilität $<20 \%$
Tab. 5b Schweregrade bei unbehandeltem Asthma (Erwachsene) mor oder Stenose im Bereich der großen Atemwege gedacht werden! Bei der numerischen Dokumentation einer extrathorakalen Stenose sind folgende Quotienten hilfreich: $\mathrm{MIF}_{50} / \mathrm{MEF}_{50}<1,0, \mathrm{FEV}_{1} / \mathrm{PEF}>10,0, \mathrm{FEV}_{1} / \mathrm{FEV}_{0,5}>1,5$. Allerdings ist $\mathrm{zu}$ beachten, dass die Sensitivität dieser Konstellationen sehr gering ist.

Reversibilitätstest mit Bronchodilatatoren: Wird eine obstruktive Ventilationsstörung vermutet, sollte ein Bronchodilatationstest durchgeführt werden. Die Messungen der $\mathrm{FEV}_{1}$ erfolgen vor und 15 Minuten nach Inhalation eines schnell wirksamen Beta2-Sympathomimetikums (z.B. bis zu $400 \mu \mathrm{g}$ Salbutamol in 4 separaten Dosen) bzw. vor und frühestens 30 Minuten nach Inhalation eines schnell wirksamen Anticholinergikums (z.B. $160 \mu \mathrm{g}$ Ipratropiumbromid). Ein Anstieg der $\mathrm{FEV}_{1}$ um mehr als $200 \mathrm{ml}$ und um mindestens 15\% des Ausgangswertes belegt die (Teil-) Reversibilität der Bronchialobstruktion. Die Messung der Reaktion der Atemwegsobstruktion auf Bronchodilatatoren ist vor allem zur Differenzialdiagnose zwischen Asthma und COPD notwendig: Je höher der Grad der Reversibilität, umso wahrscheinlicher ist die Diagnose Asthma bronchiale. Bei COPD wird die nach Inhalation von Bronchodilatatoren bestimmte $\mathrm{FEV}_{1}$ zur Schweregradeinteilung verwendet. Bei der Beurteilung der Reversibilität ist auf die vorausgegangene Karenz von Bronchodilatatoren zu achten (kurzwirksame Beta-Mimetika und Anticholinergika 4 Stunden, langwirksame Beta-Mimetika und retardierte Theophyllinpräparate 12 Stunden, langwirksame Anticholinergika 48 Stunden).

\section{Restriktive Ventilationsstörung}

Eine restriktive Ventilationsstörung ist durch eine Behinderung der normalen Lungenausdehnung charakterisiert. Definiert ist sie durch eine Verminderung der Totalkapazität, die allerdings spirometrisch nicht gemessen werden kann. Eine verminderte Vitalkapazität alleine kann nicht den Nachweis einer restrikti- ven Ventilationsstörung erbringen. Wenn der $\mathrm{FEV}_{1} / \mathrm{IVC}-\mathrm{Quo}$ tient normal oder erhöht ist, darf man allerdings bei einer verminderten Vitalkapazität eine restriktive Ventilationsstörung vermuten. Der spirometrische Schweregrad der restriktiven Ventilationsstörung ergibt sich aus der Einschränkung der IVC, ausgedrückt in \% des Sollwerts (Tab. 6). Sie ist ein pathophysiologisch sinnvolleres Maß, die behinderte Lungenausdehnung auszudrücken, wenn die TLC nicht zur Verfügung steht. Natürlich ist aber bei einer verminderten IVC auch die $\mathrm{FEV}_{1}$ erniedrigt. Man unterscheidet prinzipiell eine pulmonale von einer extrapulmonalen Restriktion, die jeweiligen Ursachen sind in - Tab. 6 zusammengefasst. Die pulmonale Restriktion ist durch eine vermehrte Steifigkeit (verminderte Compliance) der Lunge bedingt. Daher ist die „Entleerung“ der Lunge bei forcierter Exspiration durch die erhöhte Retraktionskraft beschleunigt, der Peak flow kaum erniedrigt und die Fluss/Volumen-Kurve exspiratorisch konkavbogig verformt. Beim Zustand nach Pneumektomie liegt eine restriktive Ventilationsstörung vor, wobei die Steifigkeit der verbliebenen Lungenhälfte normal ist. Bei extrapulmonaler Restriktion ist die Lungenausdehnung trotz normaler Lunge, z.B. durch Atemmuskelschwäche oder Thoraxdeformität, vermindert. Dabei ist die Fluss/Volumen-Kurve kleiner und nicht verformt. Zur Diagnostik der Atemmuskelschwäche als Ursache einer Restriktion ist die Bestimmung der maximalen Inspirationskraft notwendig (siehe Empfehlungen der Deutschen Atemwegsliga zur Messung der inspiratorischen Muskelfunktion). Die Einteilung des Schweregrades findet sich in - Tab. 6. Auch hier muss nicht der Schweregrad der Ventilationsstörung mit dem Schweregrad der Erkrankung übereinstimmen. So können z. B. Patienten mit schwerster Hypoxie bei Lungenfibrose lediglich eine leichte restriktive Ventilationsstörung aufweisen. Abb. 4. 
Definition

TLC $<5$. Perzentile des Sollwerts

\section{Schweregrade}

$\begin{array}{llll}\text { I } & \text { leicht } & \text { IVC } & >70 \% \text { Soll } \\ \text { II } & \text { mäßig } & \text { IVC } & 60-69 \% \text { Soll } \\ \text { III } & \text { mittelschwer } & \text { IVC } & 50-59 \% \text { Soll } \\ \text { IV } & \text { schwer } & \text { IVC } & 35-49 \% \text { Soll } \\ \text { V } & \text { sehrschwer } & \text { IVC } & <35 \% \text { Soll } \\ \text { Differenzialdiagnosen } & & & \end{array}$

a) pulmonal

diffuse Lungenparenchymerkrankungen

b) extrapulmonal

Pneumothorax

Silikose

Pneumonie

Pneumonitis

zystische Fibrose (Mukoviszidose)

Bronchiektasie

Linksherzinsuffizienz (oft mit Obstruktion)
Atemmuskelschwäche (z. B. neuromuskuläre Erkrankungen, Myopathien, Steroide, Hyper/Hypothyreose etc.)

Kyphoskoliose

instabiler Thorax

Pleuraerguss, -schwarte

Zwerchfellparese

Adipositas
Tab. 6 Restriktive Ventilationsstörung

c) Zustand nach Pneumektomie

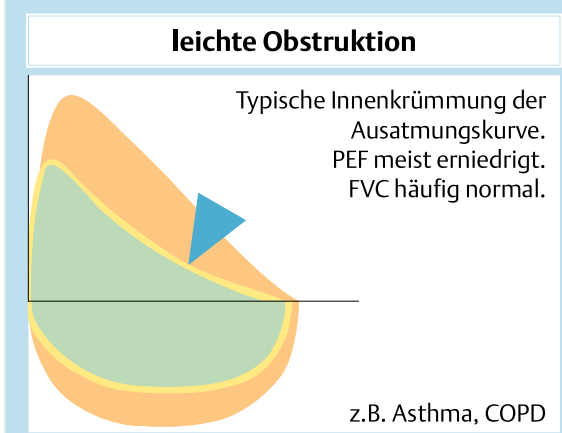

$4 a$

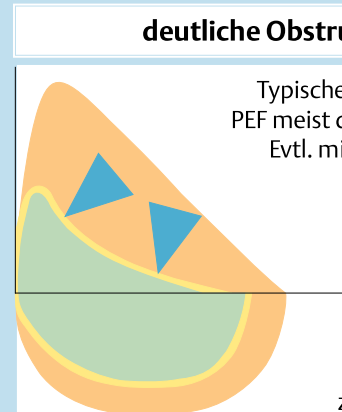

z.B. Asthma, COPD

$4 b$

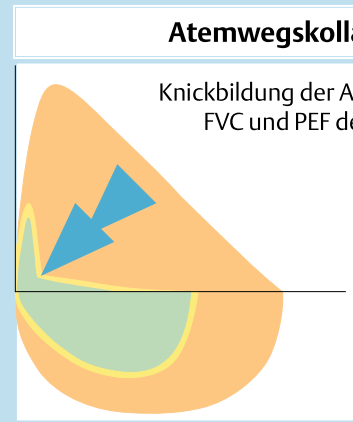

z.B. Emphysem

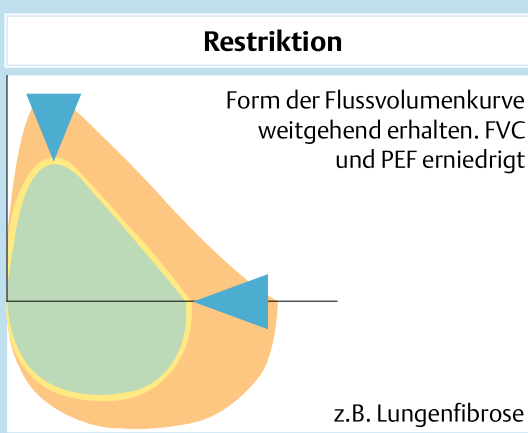

4d variable extrathorakale Stenose

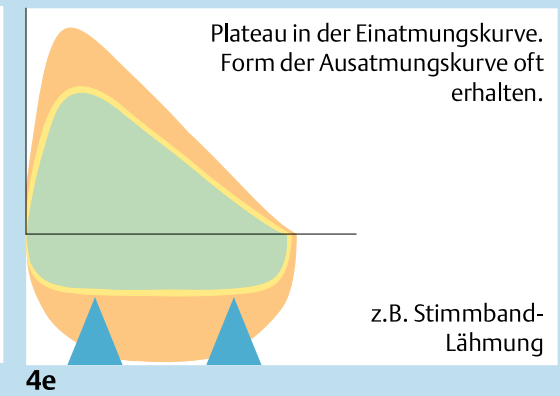

variable intrathorakale Stenose

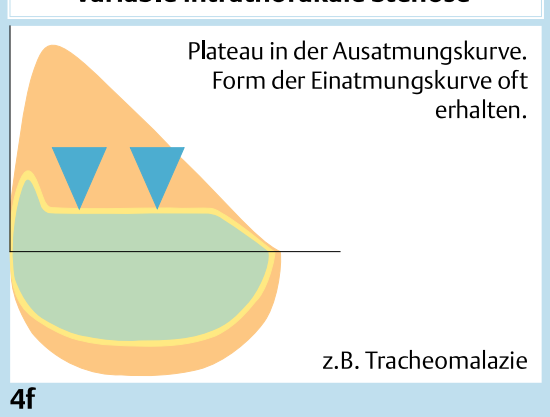

Abb. 4 Krankheitszeichen.

3. Differenzialdiagnose bei verminderter Vitalkapazität Eine verminderte Vitalkapazität ist bei verminderter Totalkapazität Ausdruck einer restriktiven Ventilationsstörung, bei normaler oder erhöhter Totalkapazität ist sie Folge einer Lungen- überblähung. Erfahrungsgemäß ist bei einer obstruktiven Ventilationsstörung mit einem $\mathrm{FEV}_{1} /$ IVC-Quotienten unter $55 \%$ die Verminderung der Vitalkapazität fast immer durch eine Lungenüberblähung bedingt. Der früher häufig verwendete Terminus 


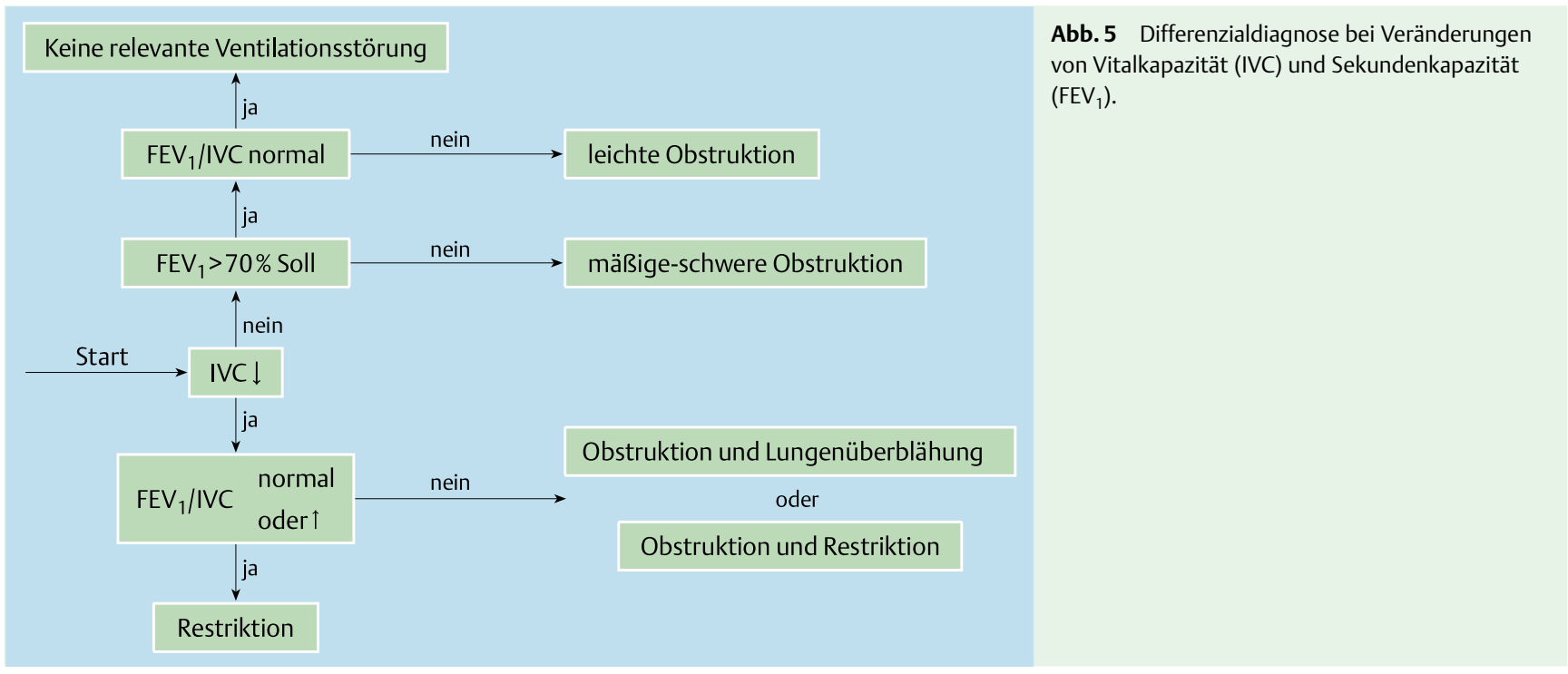

„kombinierte Ventilationsstörung“ für die Kombination aus eingeschränktem $\mathrm{FEV}_{1} /$ IVC-Quotienten und verminderter Vitalkapazität unterschied nicht zwischen den Differenzialdiagnosen Restriktion vs. Lungenüberblähung und sollte wegen dieser Ungenauigkeit nicht mehr verwendet werden. Wenn z.B. bei einer restriktiven Ventilationsstörung bei Lungengerüsterkrankung der $\mathrm{FEV}_{1} /$ IVC-Quotient durch gleichzeitig bestehende Obstruktion (z. B. bei Sarkoidose II und III) vermindert ist, sollte eine simultan bestehende restriktive und obstruktive Ventilationsstörung diagnostiziert werden (Abb. $\bigcirc 5$ ).

\section{Danksagung}

Grafiken aus www.spiro-webCard.de. Wir danken Frau Dr. U. Butt für die redaktionelle Bearbeitung und Frau Barbara Criée für die grafische Bearbeitung.

\section{Literatur}

Diese Empfehlungen wurden an die offiziellen gemeinsamen Empfehlungen der American Thoracic Society und der European Respiratory Society (ATS/ERS Task Force: Standardisation of lung function testing, Eur. Respir. J. 26:2005) angeglichen. Allerdings wurde für die Schweregradeinteilung der restriktiven Ventilationsstörung die IVC verwendet (s. Text).

\section{Anhang l: Hygieneempfehlungen}

Insbesondere bei Verwendung von Geräten, bei denen auch inspiratorische Atemmanöver durchgeführt werden, sind Vorsichtsmaßnahmen zu beachten, da der Patient u.U. direkt gefährdet ist:

Bei Ausstattung mit beheiztem Pneumotachographen-Sieb ist davon auszugehen, dass die meisten Keime innerhalb kur- zer Zeit abgetötet werden. Wenn Nasenklemme, Mundstück und Krümmer nach jeder Messung gewechselt werden, ist das Risiko einer Infektion mit pathogenen Keimen zu vernachlässigen. Bei unbeheizten Systemen sollte nach jedem Patienten sicherheitshalber der gesamte Messkopf gewechselt oder der Einsatz von Atemfiltern erwogen werden.

Bei erhöhtem Risiko (Hepatitis B oder C, Mukoviszidose, offener Lungen-Tuberkulose, AIDS, Immundefizienz, immunsuppressiver oder zytostatischer Therapie) wird anschließend entweder ein frischer Pneumotachograph verwendet bzw. können Einwegfilter entsprechend den Empfehlungen der Gerätehersteller verwendet werden. Damit wird ein verlässlicher Schutz gegen Bakterien und Viren gewährleistet, ohne dass die Messergebnisse in relevantem Ausmaß beeinträchtigt werden.

Zum Schutz des Personals und der nachfolgenden Patienten sollte nach jeder Untersuchung eines Patienten u.a. eine sorgfältige Reinigung und Desinfektion der Hände erfolgen.

Eine weitere Sicherheitsmaßnahme besteht darin, Messungen an infektiösen Patienten am Schluss der Lungenfunktionsuntersuchungen innerhalb eines Tages bzw. im Krankenzimmer durchzuführen.

Damit ergeben sich folgende Minimalanforderungen:

Demontage von Nasenklemme, Mundstück und gegebenenfalls Krümmer nach jedem Patienten, bei unbeheiztem Pneumotachographen wird dieser ebenfalls demontiert (bzw. das stattdessen eingesetzte widerstandsarme Einwegfilter). Sorgfältige Reinigung und Desinfektion der Hände (Kontamination!).

Montage von Krümmer, neuem Mundstück und Nasenklemme (bzw. des neuen Pneumotachographen oder Einwegfilters).

Je nach Räumlichkeit und Untersuchungsaufwand kann eine Lüftung des Lungenfunktionslabors nach jedem Patienten sinnvoll sein. 